\title{
The "No-Reflow" Phenomenon after Temporary Coronary Occlusion in the Dog
}

\author{
Robert A. Kloner, Charles E. Ganote, and Robert B. Jennings \\ From the Department of Pathology, Northwestern University Medical School, \\ Chicago, Illinois 60611
}

\begin{abstract}
A BSTRACT The role of microvascular damage in the genesis of the "no-reflow" phenomenon was investigated in the left ventricular myocardium of dogs subjected to temporary occlusions of a major coronary artery for 40 and $90 \mathrm{~min}$. Intravenous carbon black or thioflavin S (a fluorescent vital stain for endothelium) were used to demonstrate the distribution of coronary arterial flow in control and damaged myocardium. These tracers were injected simultaneously with release of the coronary occlusion or after 5 or $20 \mathrm{~min}$ of reflow of coronary arterial blood. After $40 \mathrm{~min}$ of ischemia plus arterial reperfusion, usually the tracers were evenly distributed throughout the damaged tissue at each time of reperfusion. On the other hand, when reflow was allowed after $90 \mathrm{~min}$ of ischemia, portions of the inner half of damaged myocardium were not penetrated by the tracers. Electron microscopic study of this poorly perfused tissue revealed severe capillary damage; endothelial cells with large intraluminal protrusions and decreased pinocytic vesicles were common. Also, occasional intraluminal fibrin thrombi were noted, as well as extravascular fibrin deposits and erythrocytes. Myocardial cells were swollen in both poorly perfused and well-perfused irreversibly injured tissue. Contraction bands and mitochondrial $\mathrm{Ca}^{2+}$ accumulation were prominent features of irreversible injury with reflow at 40 min but were not noted after $90 \mathrm{~min}$ of ischemia in areas with poor perfusion. These results suggest that $40 \mathrm{~min}$ of ischemia were tolerated by the capillary bed of the dog heart without serious capillary damage or perfusion defects, but that $90 \mathrm{~min}$ of ischemic injury was associated with the "no-reflow" phenomenon, i.e., failure to achieve uniform reperfusion. This failure of reflow was associated with extensive capillary damage and myocardial cell swelling. Death of severely ischemic myo-
\end{abstract}

Mr. Kloner was a predoctoral fellow during the time of this study (National Institutes of Health grant GM 00131).

Received for publication 3 June 1974 and in revised form 19 August 1974 cardial cells in this model occurs before the onset of capillary damage and the no-reflow phenomenon.

\section{INTRODUCTION}

Recent advances, such as coronary bypass surgery and the development of fibrinolytic agents, eventually may make it possible to release coronary occlusions during the acute stage of myocardial injury. However, several investigators have suggested that, under some circumstances, restoration of arterial flow into the previously ischemic tissue either does not occur or is greatly impeded. This so-called "no-reflow" phenomenon has been demonstrated in kidney $(1,2)$, brain $(3-6)$, and skin (7). Moreover, Krug, de Rochemont, and Korb (8) have suggested that this phenomenon occurs in the cat heart. In this tissue they have shown that significant portions of the inner myocardium cannot be perfused after temporary occlusions of 60-120 min. Also, the no-reflow phenomenon has been suspected in canine myocardium (9), but direct data supporting this conclusion have not been reported.

Extensive study of the acute changes occurring in myocardium irreversibly injured by ischemia during the first few minutes after the onset of reflow have shown that the damaged cells accumulate massive quantities of $\mathrm{Ca}^{2+}$ from the plasma reperfusing the tissue $(10,11)$. These observations were made after $40 \mathrm{~min}$ of ischemic injury in the dog heart and indicate that significant reflow did occur after this period of ischemia. The experiments presented in this paper, however, establish that a no-reflow phenomenon does occur with severe ischemia of longer duration. Our experiments were designed to characterize this no-reflow phenomenon. We aimed to determine the time at which no reflow was detectable as well as the nature of the pathologic changes occurring in the poorly perfused tissue. We have shown that no reflow does occur in parts of the severely ischemic myocardium after 90 but not after $40 \mathrm{~min}$ of ischemia and 

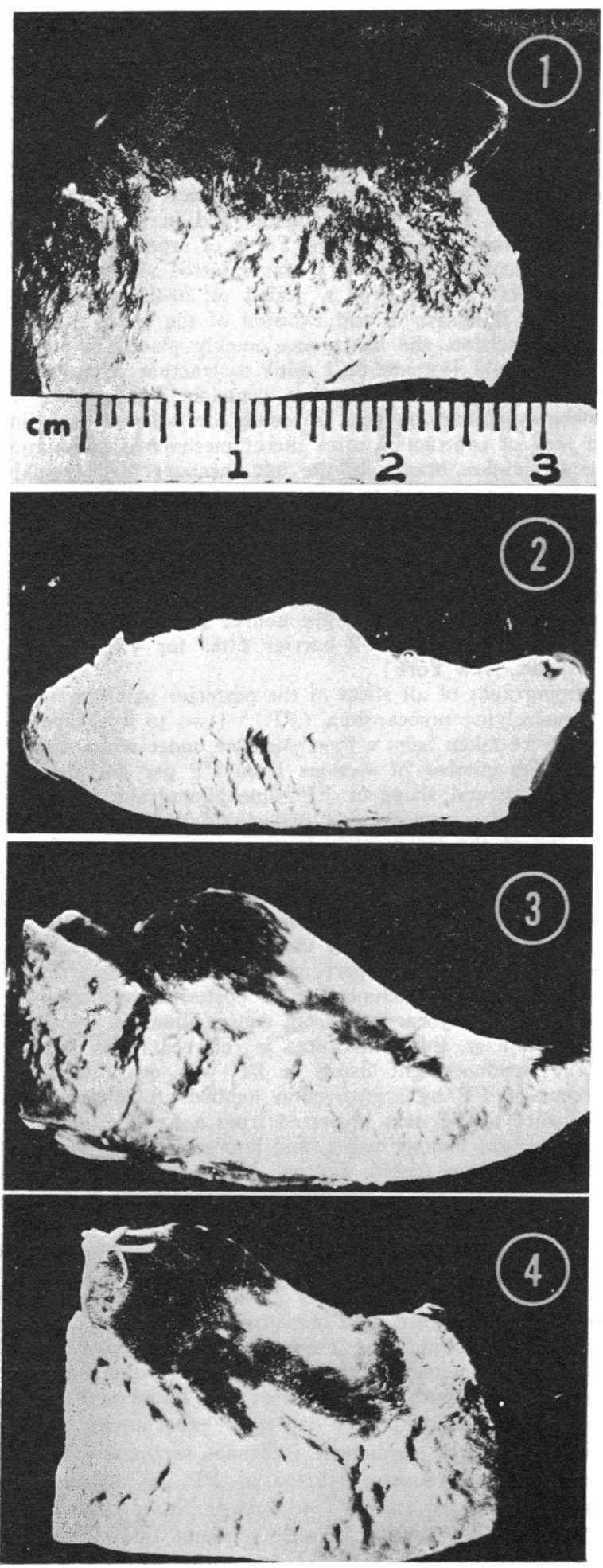

FIgURE 1 Posterior papillary muscle and underlying myocardium (PP) permanently ischemic for $90 \mathrm{~min}$. The subepicardium (bottom of picture) is homogeneously fluores- is associated with extensive damage to the microvasculature.

\section{METHODS}

Experimental design. Distribution of arterial flow to the myocardium of the dog was estimated by observing the distribution of thioflavin $\mathrm{S}$ injected $10 \mathrm{~s}$ before excision of the heart, according to techniques described previously (12). Thioflavin $\mathrm{S}$ is a fluorescent vital dye for endothelium. Capillaries receiving flow will fluoresce brightly when viewed under UV light. Carbon black also was used to trace distribution of coronary flow.

Dogs were divided into three groups: (a) Permanent ischemia group; four dogs were killed after $90 \mathrm{~min}$ of permanent ischemia. (b) Transient ischemia group; all dogs in this group received either 40 or $90 \mathrm{~min}$ of ischemia and variable periods of reperfusion induced by quickly opening the occlusion. Within a few seconds after reperfusion, the portion of the epicardial surface of the heart which previously had been cyanotic now appeared hyperemic. 12 dogs were killed after $40 \mathrm{~min}$ of temporary ischemia with either $10-12 \mathrm{~s}$ of reperfusion (one dog), 5 min of reperfusion (seven dogs), or after $20 \mathrm{~min}$ of reperfusion (four dogs). 15 dogs were killed after $90 \mathrm{~min}$ of temporary ischemia with either 10-12 s of coronary reperfusion (four dogs), $5 \mathrm{~min}$ of reperfusion (seven dogs), or with $20 \mathrm{~min}$ of reperfusion (four dogs). (c) Two dogs were killed after sham operations in which the chest was opened and the artery was isolated but not occluded.

Experimental animals. 57 adult mongrel dogs of both sexes, weighing 21-50 lb, were housed in air-conditioned quarters maintained at $70^{\circ} \mathrm{F}$ and given free access to Borden's dog chow (Borden Inc., New York) and water. They were fasted overnight before use.

Operative procedure. Dogs were anesthetized with 30 $\mathrm{mg} / \mathrm{kg}$ body wt sodium pentobarbital (Diabutal, Diamond Laboratories Inc., Des Moines, Iowa). Additional pentobarbital was given as needed to maintain inhibition of the corneal reflex. The dogs were intubated and ventilated with a Harvard respirator (model 1063, Harvard Apparatus Co., Inc., Millis, Mass.) at about $300 \mathrm{ml}$ of room air $/ \mathrm{kg}$ body wt $/ \mathrm{min}$. Arterial blood pressure recordings were obtained from a right femoral artery catheter and recorded on a Grass polygraph (model 5P1, Grass Instrument Co., Quincy, Mass.). Continuous electrocardiographs from standard limb leads were recorded on the polygraph. The chest was opened through the fourth left intercostal space, the lungs gently retracted, and pericardium widely excised to expose the heart. The circumflex branch of the left coronary artery was dissected free of surrounding fat and occluded close $(5-15 \mathrm{~mm})$ to its origin with a Goldblatt clamp. Dogs which demonstrated cyanosis on the posterolateral wall of the left ventricle and S-T seg-

cent. Portions of the midmyocardium and most of the subendocardium (top of picture) are nonfluorescent.

FIGURE $2 \mathrm{PP}$ temporarily ischemic for $40 \mathrm{~min}$ with $5 \mathrm{~min}$ of coronary reperfusion. The myocardium fluoresces homogeneously.

FIGURE 3 PP temporarily ischemic for $90 \mathrm{~min}$ with $5 \mathrm{~min}$ of coronary reperfusion. Portions of the subendocardium are nonfluorescent and thus represent areas of no reflow. FIGURE 4 PP temporarily ischemic for $90 \mathrm{~min}$ with 20 min of coronary reperfusion. Portions of the subendocardium and inner midmyocardium are nonfluorescent. 


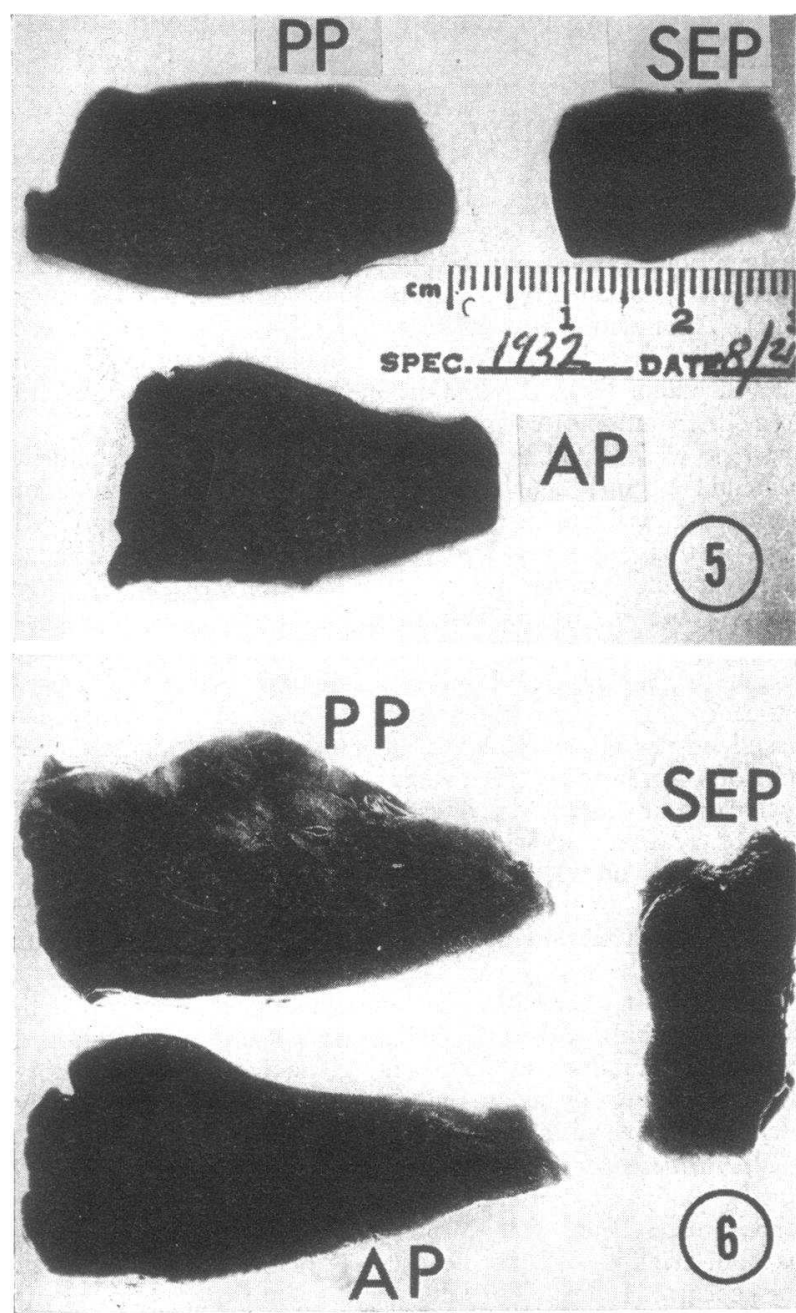

FIgURE $5 \mathrm{PP}$, which was temporarily ischemic for $40 \mathrm{~min}$ with $5 \mathrm{~min}$ of reperfusion, is homogeneously perfused with carbon black. AP (anterior papillary muscle and underlying myocardium) and a slice of anterior interventricular septum (SEP) are also dark black.

FIGURE 6 PP temporarily ischemic for $90 \mathrm{~min}$ with $5 \mathrm{~min}$ of coronary reperfusion. Patches in the subendocardium of PP are not perfused with carbon black. AP and septum (SEP) are dark black.

ment elevation in leads II, III, and AVf were used for this study.

Of the original 57 dogs, 24 died from ventricular fibrillation either during occlusion or upon reflow. All surviving dogs used in this study were killed by excision of the heart.

Preparation of thioflavin $S$ and carbon black. Thioflavin $S$ was obtained from Pfaltz \& Bauer, Inc. (Flushing, N. Y.). It was prepared fresh as a $4 \%$ solution in normal saline. Before injection, particulate matter was removed from the dye: by ultracentrifugation $(16,000 \mathrm{rpm})$ for $5 \mathrm{~min}$.

Pelikan' carbon black (20-30-nm carbon particles) was obtained from John Henschel \& Co., Inc. (Farmingdale,
N. Y.) as a $10 \%$ suspension. It was thoroughly mixed before injection.

Evaluation of coronary flow distribution. To study the transmural distribution of coronary flow, dogs were given $1.0 \mathrm{~cm}^{3} / \mathrm{kg}$ body $\mathrm{wt}$ of a $4 \%$ solution of thioflavin $S$ in normal saline, via the saphenous vein. The dye was injected by hand over a period of 6-8 s, $10 \mathrm{~s}$ before excision of the heart. In addition, in three dogs receiving $40 \mathrm{~min}$ of ischemia and $5 \mathrm{~min}$ of reperfusion, and in three dogs receiving $90 \mathrm{~min}$ of ischemia and $5 \mathrm{~min}$ of reperfusion, carbon black suspension $\left(3 \mathrm{~cm}^{3} / \mathrm{kg}\right)$ was injected slowly by hand into the left atrium over a period of 20-25 $\mathrm{s}$ before injection of thioflavin $\mathrm{S}$ and excision of the heart.

After excision, the heart was quickly placed in $500-750$ $\mathrm{ml}$ of ice-cold isotonic $\mathrm{KCl}$ until contraction ceased (usually within $30-40 \mathrm{~s}$ ). The left ventricle was opened and posterior papillary muscle inspected for paleness, swelling, and lack of contraction after direct mechanical stimulation. The circumflex branch of the left coronary and its major branches were opened to detect any thrombi. Then the left ventricle was sectioned into $0.2-\mathrm{cm}$ longitudinal strips. Heart slices were examined for carbon black distribution in white light and for thioflavin S fluorescence under UV light, using a high intensity mercury lamp (E. Leitz, Inc., Rockleigh, N. J.) as a light source and a Zeiss BG 12 excitation filter and Y 2 barrier filter for viewing (Carl Zeiss, Inc., New York).

Photographs of all slices of the posterior papillary muscle and underlying myocardium $(P P)^{1}$ (two to five slices per dog) were taken from a fixed distance under white and UV light. The number of sections from PP per dog depended on the size and shape of PP. One photograph of the anterior papillary muscle and underlying myocardium (AP) and one of the interventricular septum were taken from a fixed distance.

Assessment of flow distribution in PP was based on measurements within an anatomic area defined by the posterior papillary muscle and underlying myocardium, within the boundaries of two perpendicular lines drawn from the upper and lower attachment of the posterior papillary muscle fibers on the myocardium from endocardium to epicardium. For simplicity, this entire area is referred to as PP.

The nonfluorescent tissue in PP was quantitated as a percent of $\mathrm{PP}$ by a projection method. A photograph of each slice of PP was projected from a fixed distance onto heavy-weight biology paper, and fluorescent and nonfluorescent areas were traced, cut out, and weighed. The overall nonfluorescent area of PP was determined from the average of these slices.

Light and electron microscopy. One slice from the center of PP was fixed in $10 \%$ aqueous formalin buffered with phosphate to $\mathrm{pH}$ 7.0. After paraffin embedding, sections were cut and stained with hematoxylin and eosin. To determine what morphologic changes might contribute to the no-reflow phenomenon, tissue for electron microscopy was sampled from both nonfluorescent and fluorescent areas of $\mathrm{PP}$ in $90-\mathrm{min}$ temporary ischemia experiments. In dogs with $90 \mathrm{~min}$ of permanent ischemia, sections were taken from pale nonfluorescent areas of PP. In addition, nonischemic control tissue was taken from the anterior interventricular septum. Tissue was cut into $1-\mathrm{mm}$ cubes under cold phosphate-buffered $1 \%$ osmic acid and fixed for

\footnotetext{
${ }^{1}$ Abbreviations used in this paper: AP, anterior papillary muscle and underlying myocardium; $\mathrm{PP}$, posterior papillary muscle and underlying myocardium.
} 


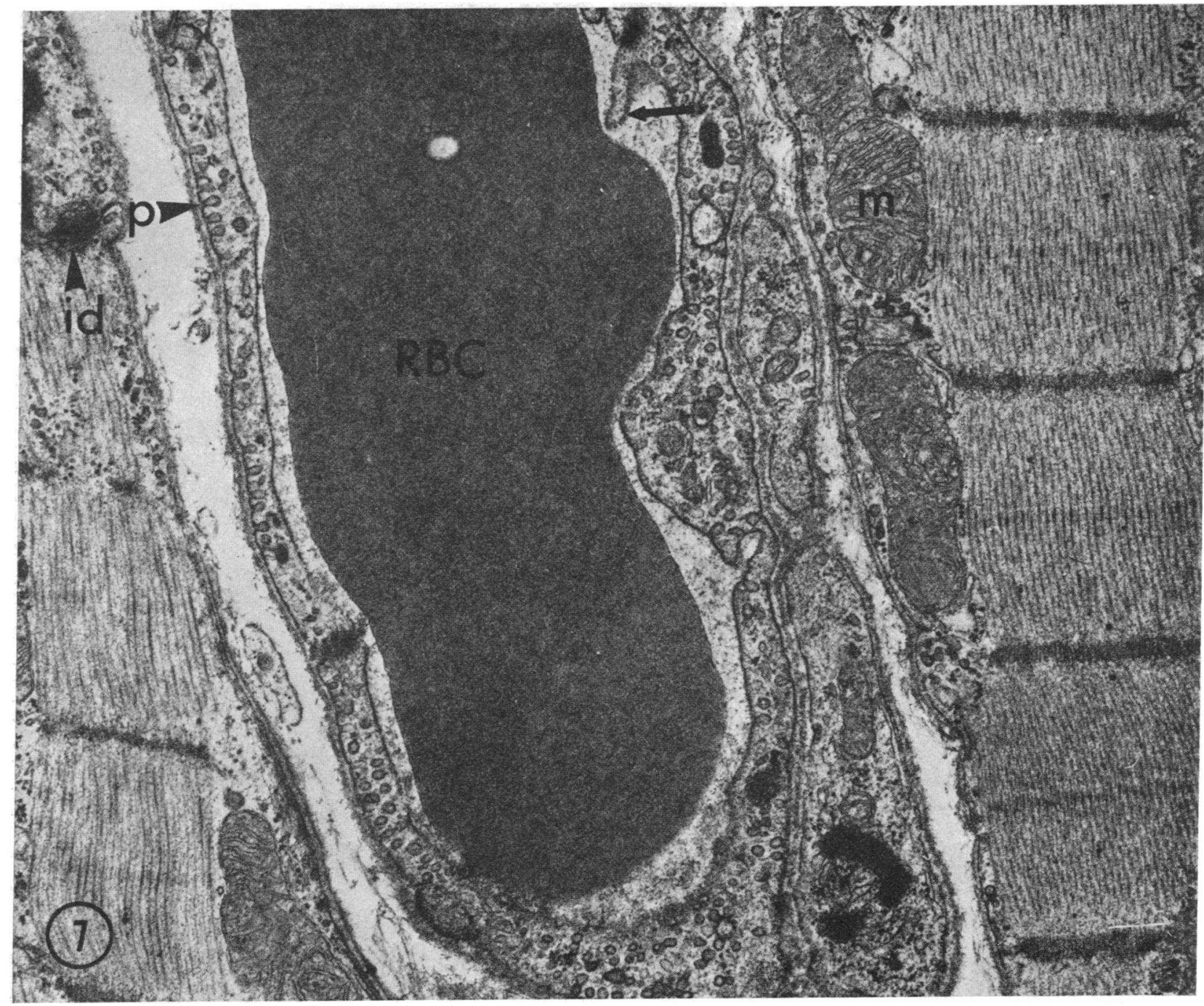

FIGURE 7 Nonischemic control myocardium from interventricular septum. Capillary endothelium is thin and pinocytotic vesicles (P) are numerous. A short endothelial fold is present (arrow). Mitochondrial cristae are tightly packed. $\mathrm{RBC}$, red blood cell; $\mathrm{m}$, mitochondria; id, intercalated disk. $\times 24,000$.

$60 \mathrm{~min}$ at $5^{\circ} \mathrm{C}$. After fixation the cubes were dehydrated at room temperature in graded alcohols, passed through propylene oxide and placed in a mixture of $1: 1$ propylene oxide and Epon 812 (Shell Chemical Co., New York) overnight before embedding in Epon 812. Sections were cut on a Porter-Blum MT-2 ultramicrotome using glass or diamond knives. Sections approximately $0.5-\mu \mathrm{m}$ thick were stained with toluidine blue for light microscopy. Thin sections mounted on copper grids, stained with $7.7 \%$ aqueous uranyl acetate and/or $0.3 \%$ lead citrate, or unstained, were examined in a Hitachi HU-12 (Hitachi Ltd., Tokyo, Japan) or a Philips EM-200 electron microscope (Philips Electronic Instruments, Mount Vernon, N. Y.).

Electron microscopic findings of damaged and control tissue after $40 \mathrm{~min}$ of ischemia and $2,5,10$, and $20 \mathrm{~min}$ of arterial reperfusion have recently been reported (13).

\section{RESULTS}

\section{Coronary flow distribution}

\section{SHAM-OPERATED DOGS}

All heart slices from sham-operated dogs demonstrated an homogeneous yellow-green fluorescence when examined under ultraviolet light.

\section{Permanent CORONARY OCCLUSIONS}

Large portions of PP were nonfluorescent in all dogs with $90 \mathrm{~min}$ of permanent ischemia. The subendocardium (inner one-third of myocardium) was always nonfluores- 

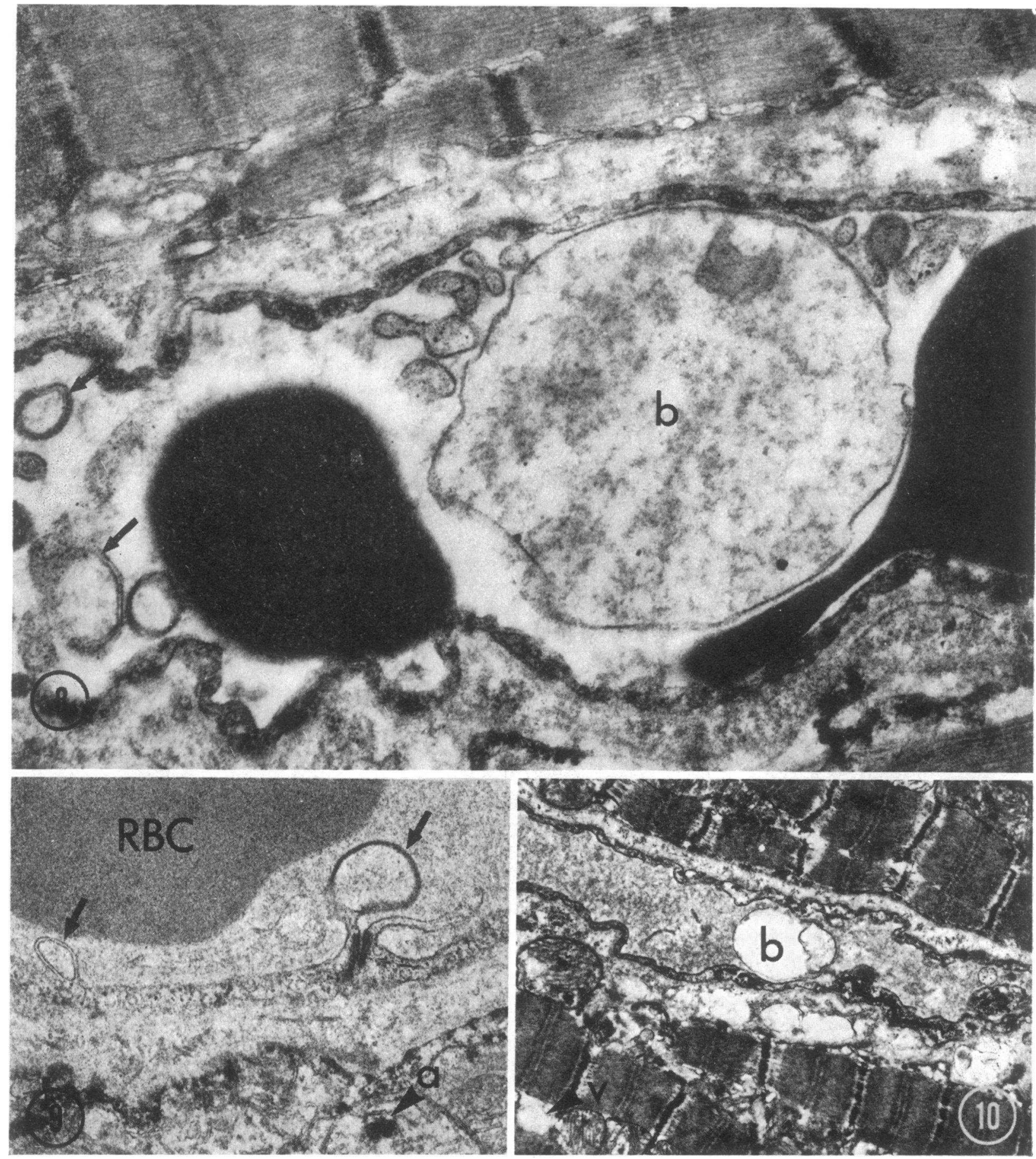

FIGURE 8 PP permanently ischemic for $90 \mathrm{~min}$. Virtually no pinocytotic vesicles are present in the capillary endothelium. A large membrane-bound body (b) and several smaller ones (arrows) are present in the capillary lumen. $\times 21,600$.

FIGURE 9 PP permanently ischemic for $90 \mathrm{~min}$. Two swollen endothelial protrusions (arrows) appear to be budding off of the intraluminal surface. One is in close approximation to a tight junction. Few pinocytotic vesicles are present. A large amorphous dense body (a) is present in a swollen mitochondrion. $\mathrm{RBC}$, red blood cell. $\times 28,000$.

FIGURE 10 PP permanently ischemic for $90 \mathrm{~min}$. I bands are prominent. Mitochondria are swollen with disrupted cristae and amorphous matrix densities. Intermyofibrillar edema and a vacuole (v), possibly representing dilated sarcoplasmic reticulum, are present. $\Lambda$ membrane-bound body (b) is floating in capillary devoid of pinocytotic vesicles. $\times 8,350$. 
cent. The midmyocardium (middle one-third) was often nonfluorescent, but the subepicardium (outer one-third) was seldom nonfluorescent (Fig. 1).

The percent of nonfluorescent tissue in $\mathrm{PP}$ was $49 \pm$ $10 \%$. This was similar to the percent of nonfluorescent tissue in $\mathrm{PP}$ in previous studies with 15 and $60 \mathrm{~min}$ of permanent ischemia (50 and 48\%, respectively) (12).

Sections of left ventricle taken between $\mathrm{PP}$ and AP always had large areas of nonfluorescence in the inner two-thirds of myocardium. The tip of AP was often nonfluorescent. However, the nonischemic anterior interventricular septum was always homogeneously fluorescent and thus served as an internal control.

\section{TEMPORARY CORONARY OCCLUSIONS}

In one dog with $10-12 \mathrm{~s}$ of reperfusion and in seven dogs with $40 \mathrm{~min}$ of temporary ischemia and $5 \mathrm{~min}$ of coronary reperfusion, the $\mathrm{PP}$ was homogeneously fluorescent (Fig. 2). Also, three out of four dogs with $40 \mathrm{~min}$ of ischemia and $20 \mathrm{~min}$ of reperfusion demonstrated homogeneous fluorescence in PP. In the fourth dog, in which the PP was extremely swollen compared with the other dogs, $4 \%$ of the PP was nonfluorescent, indicating that this area was not well perfused at the time the dye was injected. This area of poor perfusion was confined to the subendocardium in the projecting part of the PP. In all dogs with 40 or $90 \mathrm{~min}$ of temporary coronary occlusion, the nonischemic anterior interventricular septum was always homogeneously fluorescent.

In all dogs receiving $90 \mathrm{~min}$ of temporary ischemia with $10-12 \mathrm{~s}, 5$, or $20 \mathrm{~min}$ of coronary reperfusion, portions of the subendocardium and sometimes of the inner midmyocardium of PP demonstrated areas of nonfluorescence (Figs. 3 and 4). Surrounding these areas of nonfluorescence, a zone of fluorescence brighter than nonischemic control tissue could sometimes be distinguished. The subepicardial third of myocardium always was homogeneously fluorescent, suggesting that significant reflow had occurred into this region. $11 \pm 2 \%$ of $\mathrm{PP}$ was nonfluorescent in those dogs receiving 10-12 s of coronary reperfusion; $12 \pm 4 \%$ of $\mathrm{PP}$ was nonfluorescent in those dogs receiving $5 \mathrm{~min}$ of reperfusion; and $10 \pm 3 \%$ of $\mathrm{PP}$ was nonfluorescent in those dogs receiving $20 \mathrm{~min}$ of reperfusion. Sections taken between PP and AP and from AP, especially the tip of AP, often had small areas of subendocardial nonfluorescence. Presumably because of quenching, the overall intensity of thioflavin S fluorescence was decreased in dogs which also had received carbon black, but the fluorescent stain was still adequate to assess distribution of arterial flow.

In the three dogs which received $40 \mathrm{~min}$ of ischemia and $5 \mathrm{~min}$ of reperfusion (Fig. 5), all heart slices were uniformly dark black when injected with carbon black. In dogs receiving $90 \mathrm{~min}$ of ischemia and $5 \mathrm{~min}$ of re- flow, pink patches into which the carbon black did not penetrate were present in portions of the subendocardium and inner midmyocardium of $\mathrm{PP}$ and sections between PP and AP (Fig. 6). These pink areas corresponded closely to the zones of nonfluorescence seen with UV light. The nonischemic control anterior interventricular septum was always homogeneously black in color.

Gross thrombi were not present in either the circumflex branch of the left coronary artery or any of its major branches.

There was a slight but significant fall in mean arterial blood pressure after circumflex occlusion (128$118 \mathrm{~mm} \mathrm{Hg}$ ) in dogs with both 40 and $90 \mathrm{~min}$ of ischemia. Blood pressure remained at this slightly lower level throughout and after release of the occlusion. Arterial pressures during and after the release of the occlusion were similar in both 40 - and 90 -min groups.

\section{Morphologic findings}

\section{NONISCHEMIC CONTROL MYOCARDIUM}

Capillaries were intact and lumens usually contained an amorphous granular precipitate. Intraluminal folds or projections of endothelial cytoplasm were short or absent. Pinocytotic vesicles were numerous. In general, the myofibers appeared similar to previous descriptions of normal myocardium $(14,15)$ (Fig. 7).

\section{MYOCARDIUM PERMANENTLY ISCHEMIC FOR 90 MIN}

Myocardial fine structure at $90 \mathrm{~min}$ was similar to that described previously after $60 \mathrm{~min}$ of ischemia (15). However, most of the changes were more severe. In particular, capillary changes were striking. Most showed swollen intraluminal endothelial protrusions (Fig. 9). Large, clear, intraluminal membrane-bound bodies (Figs. 8 and 10) which appeared to be freely-floating were common. They were identical to those which were attached to the endothelium and were either detached from the intraluminal surface or were attached at a site not included in the plane of section. Other than these localized protrusions, the endothelium was only occasionally diffusely swollen. Endothelial cell pinocytotic vesicles were greatly decreased in number or absent, and endothelial nuclear chromatin margination and clumping were prominent. Occasionally the endothelial cells were separated, leaving spaces covered only by the basement membrane. Infrequently, small fibrin tactoids were present in arterioles and capillaries.

Myocardial cells were markedly swollen and contained cytoplasmic membrane-bound vacuoles. The sarcolemmal membrane often was lifted off of the myofilaments forming subsarcolemmal blebs. I bands were prominent (Fig. 10). Mitochondria appeared swollen with disrupted cristae, and they contained one or more large amorphous matrix densities (Figs. 9 and 10) in 


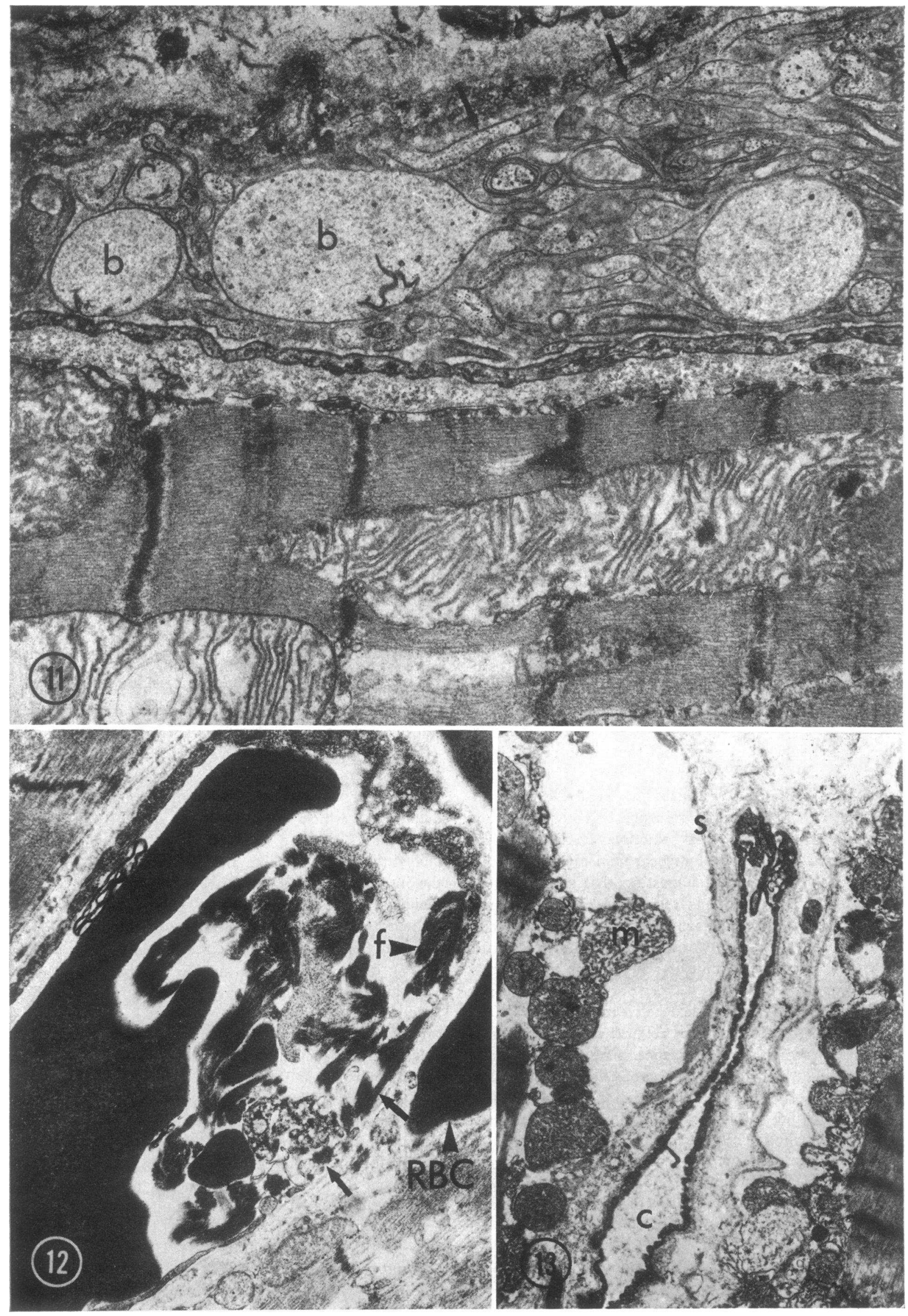


any given profile. Glycogen was virtually absent and portions of the sarcoplasmic reticulum were dilated. Nuclear chromatin clumping and margination were prominent.

\section{MYOCARDIUM TEMPORARILY ISCHEMIC FOR 90 MIN}

Areas of nonfluorescence. In sections of myocardium temporarily ischemic for $90 \mathrm{~min}$ with $10-12 \mathrm{~s}$ of coronary blood reperfusion, areas of nonfluorescence and, thus no reflow, showed changes similar to those seen after $90 \mathrm{~min}$ of permanent ischemia (Fig. 11). In addition, many capillaries contained masses of tightly packed red blood cells. Extravascular red blood cells also were observed. Endothelial protrusions and intraluminal membrane-bound bodies often filled the capillaries to a point that the lumen was obliterated (Fig. 11).

In sections of myocardium temporarily ischemic for 90 min with $5-20 \mathrm{~min}$ of reperfusion, areas of nonfluorescence were again similar to those seen after $90 \mathrm{~min}$ of permanent ischemia. Myofibrils were relaxed and mitochondria contained amorphous matrix densities. Glycogen was absent and the nuclear chromatin was aggregated peripherally. These areas of low or no flow also showed endothelial protrusions and decreased pinocytotic vesicles. In addition, however, capillaries frequently had large gaps in the endothelium which were often associated with adjacent fibrin and platelet thrombi (Fig. 12). Occasionally, capillaries appeared compressed by adjacent subsarcolemmal blebs (Fig. 13) and in others, red blood cells were packed tightly together (Fig. 14). Extravascular red blood cells were numerous in the interstitial space (Fig. 12) which also contained fibrin tactoids and myocardial cell mitochondria and membranous debris (Fig. 15).

Areas of fuorescence. In tissue sampled from fluorescent areas adjacent to zones of no reflow, capillaries appeared similar to controls except for a decrease in the number of pinocytotic vesicles and occasional endothelial nuclear chromatin clumping. There were few or no intraluminal projections, and those which were present were small. No fibrin or platelet thrombi were seen, and red blood cells were confined to the intravascular space.

Myocardial cells in areas with intact circulation showed marked architectural disorganization caused primarily by massive swelling and by the formation of large contraction bands. These bands involved 3-10 sarcomeres and obliterated myofibrillar structure. Myofibrils between contraction bands were disoriented, partially disrupted and often had thickened $Z$ bands. The swelling was characterized by an increased separation of myofibrils, by intracellular vacuoles and swollen mitochondria. Large subsarcolemmal blebs also were prominent. Mitochondria were swollen, cristae were disrupted and amorphous matrix densities were striking (Fig. 16). In contrast to previous studies with $40 \mathrm{~min}$ of ischemia and 2-20 min of blood reflow, no granular intramitochondrial dense bodies of the type associated with calcium phosphate accumulation were seen (Fig. 17).

In fluorescent tissue sampled from the outer half of myocardium which had been ischemic, little morphologic evidence of cellular injury was observed. Except for endothelial nuclear chromatin clumping and margination, the capillaries appeared similar to those of nonischemic control tissue. The myocardial cells were either normal or showed slight nuclear chromatin clumping and margination, minimal mitochondrial swelling, and occasional intracellular vacuoles.

\section{DISCUSSION}

Both thioflavin $\mathrm{S}$ and carbon black have been used in previous investigations of the no-reflow phenomenon in the kidney $(1,16)$. Thioflavin $S$ also has been used to study permanent myocardial ischemia (12), endometrial blood flow (17), and spinal cord blood flow patterns in experimental traumatic paraplegia (18). In this experiment, we used thioflavin $S$ and carbon black as tracers to measure the qualitative distribution of coronary flow after temporary ischemia. Intravenous thioflavin $S$ stains the endothelium of blood vessels which have received arterial flow between the time of injection and excision of the heart. Capillaries and large

FIGURE 11 PP temporarily ischemic for $90 \mathrm{~min}$ with $10-12 \mathrm{~s}$ of coronary blood reperfusion (from area of no reflow). Endothelial pinocytotic vesicles are sparse. The capillary lumen is full of endothelial protrusions (arrows) and membrane-bound bodies (b), some of which might represent degranulated platelets. Mitochondria are swollen with amorphous matrix dense bodies. I bands and intermyofibrillar edema are present. $\times 22,640$.

FIGURE 12 PP temporarily ischemic for $90 \mathrm{~min}$ with $20 \mathrm{~min}$ of coronary blood reperfusion (from an area of no reflow). Large gaps (arrows) are present in the capillary endothelium, with adjacent intraluminal fibrin deposits (f). An extravascular red blood cells is present $(\mathrm{RBC})$. I bands and intermyofibrillar edema are prominent. $\times 15,000$.

FIGURE $13 \mathrm{PP}$ temporarily ischemic for $90 \mathrm{~min}$ with $20 \mathrm{~min}$ of blood reperfusion (from an area of no reflow just adjacent to a fluorescent area). The sarcolemmal membrane (s) is lifted off of the myofilaments, forming a bleb-like space which appears to be compressing an adjacent capillary (c). A swollen mitochondrion (m) with disrupted cristae is present within this bleb. The presence of contracted sarcomeres suggests that some flow may have been present in this region probably in the first few seconds of reflow. $\times 6,480$. 


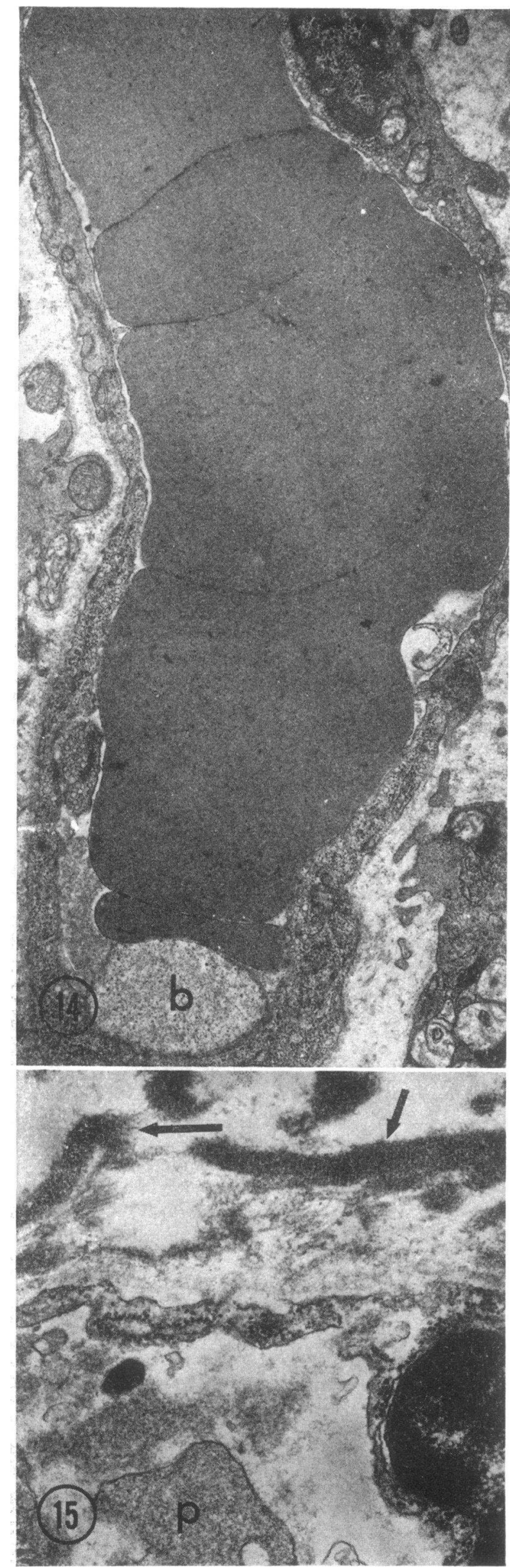

vessels which have been well perfused and stained with thioflavin $S$ fluoresce bright yellow-green when exposed to UV light. Conversely, nonfluorescent tissue represents regions of either no flow or low flow (12).

Although the use of thioflavin $\mathrm{S}$ does not allow precise quantitation of the amount of flow in an area of staining or nonstaining myocardium, it offers advantages over other qualitative techniques such as silicone rubber injections. Thioflavin $\mathrm{S}$ is water soluble and miscible with plasma; it does not produce detectable acute physiological changes or alterations in blood viscosity during the interval under study.

Evidence that the fluorescence patterns seen using thioflavin $\mathrm{S}$ have biological meaning is provided by the following observations. (a) The results obtained with thioflavin $\mathrm{S}$ are similar to those obtained with the more commonly used marker, carbon black. (b) There is a close relationship between the areas of nonfluorescence seen after permanent coronary arterial occlusion and the areas of ischemic necrosis seen grossly in the PP of dogs (12). (c) In the present study, after $90 \mathrm{~min}$ of arterial occlusion and blood reflow, the areas of fluorescence and nonfluorescence showed different ultrastructural changes. These differences in structural response are direct evidence that this technique delineated differences in regional blood flow which led to different tissue responses in the intact animal. ( $d$ ) Although we have not measured absolute myocardial blood flow in this study, we have used both tracer microspheres and thioflavin $\mathrm{S}$ in a recent study of permanent coronary occlusion. $^{2}$ In that study, blood flow in nonfluorescent areas always was reduced to at least 20 and usually $10 \%$ of normal.

In the present study, myocardium irreversibly injured by a 40 -min coronary occlusion was uniformly perfused after $0.15-0.2,5$, and $20 \mathrm{~min}$ of reflow and, although the rate of tissue perfusion was not measured, the amount of staining was equivalent to that seen in control tissue. The observation that there was uniform reflow of arterial blood after $40 \mathrm{~min}$ of ischemia agrees with previous ultrastructural (15) and electrolyte evidence that there were marked differences in the response of the injured tissue to permanent and temporary ischemia (10, 11, 19). After $40 \mathrm{~min}$ of permanent ischemia, tissue electrolytes and water remained essentially unchanged and myocardial cells were only modestly swollen (13). Allowing 2-20 min of reperfusion of coronary blood resulted in a very rapid, almost explosive, increase in tissue water, $\mathrm{Na}^{+}, \mathrm{Cl}^{-}$, and $\mathrm{Ca}^{2+}$ (19). These marked electrolyte changes could have developed only if significant blood reflow was occurring (19). Studies on the

${ }^{2}$ Kloner, R. A., K. A. Reimer, R. B. Jennings, unpublished data.

FIgURE $14 \mathrm{PP}$ temporarily ischemic for $90 \mathrm{~min}$ with 20 min of blood reperfusion (from area of no reflow). Red blood cells appear tightly packed together. $b$, intraluminal membrane-bound body. $\times 13,500$.

FIGURE 15 PP temporarily ischemic for $90 \mathrm{~min}$ with 5 min of blood reperfusion (from area of no reflow). Extravascular fibrin deposits are present (arrows). Endothelial pinocytotic vesicles are absent and nuclear chromatin is clumped. $p$, intravascular platelets. $\times 32,400$. 
source of the $\mathrm{Ca}^{2+}$ found in the damaged cells with radioactive $\mathrm{Ca}^{2+}$ confirmed that flow had occurred because almost all the increased tissue $\mathrm{Ca}^{2+}$ came from the plasma during the period of reflow (11). The fact that most capillaries appeared ultrastructurally normal after 40 min of ischemia, with or without blood reperfusion (Fig. 17) $(13,20,21)$, was further indirect evidence that the vascular system was intact and that reflow had occurred.

In contrast to the uniform staining observed in most cases after a temporary 40 -min occlusion, portions of the inner myocardium did not stain after a 90 -min coronary occlusion; this observation indicates that there was little or no reflow of arterial blood into some of the areas of maximum ischemia. Furthermore, since areas of no reflow were detected within $10-12 \mathrm{~s}$ after release of the coronary occlusion, we assume that the defect which prevented reflow was present at the end of the $90-\mathrm{min}$ episode of permanent ischemia. On the other hand, the outer myocardial wall always was fluorescent after 90 min of temporary ischemia, suggesting that it had received significant reflow.

The areas of no reflow always were smaller than the total area of ischemia identified grossly as cyanotic tissue while the vessel was occluded and were localized within the tissue identifiable grossly and microscopically as being irreversibly injured. It seems likely that, during the period under study, the no-reflow phenomenon is not the primary cause of irreversible ischemic injury of myocardial cells, since (a) myocardial cell death occurs peripheral to, as well as within areas of no reflow and, (b) irreversible myocardial cell injury is present at 40 min of ischemia, a time before the usual development of the no-reflow phenomenon.

\section{Causes of no reflow}

Endothelial changes. There are several morphologic features which appear to contribute to the no-reflow phenomenon. Although the data presented in this study do not allow us to make a definitive conclusion as to the cause of the no-reflow phenomenon, they do suggest that capillary damage plays a significant role. Capillaries in areas of no reflow consistently showed decreased numbers of pinocytotic vesicles, nuclear chromatin clumping and large protrusions of endothelial cytoplasm into the vascular lumen. Similar protrusions have been seen in cerebral ischemia by Chaing, Kowada, Ames, Wright, and Majno (3) and in several other types of cellular injury $(21,22)$. It has been suggested that the protrusions may act to occlude the capillary lumens and play a direct role in causing the no-reflow phenomenon in cerebral ischemia. Diffuse endothelial swelling has been described in the ischemic kidney (2) and may be a cause of poor reperfusion in this organ; but, diffuse endo- thelial swelling (as opposed to localized protrusions) rarely was seen in dog heart.

Since the areas of no reflow were often hyperemic and hemorrhagic and had capillaries which were packed with red blood cells, some flow must have occurred into these regions, probably during the first few seconds of reperfusion. The affected capillaries or venules were damaged by ischemia before reflow, judging from the interstitial fibrin deposits, extravascular red blood cells and large gaps in the capillary endothelium. Krug et al. (8) also have observed ruptured capillaries and hemorrhage by light microscopy in areas of no reflow in cat myocardium. Hemorrhage and microthrombi also have been noted following $3 \mathrm{~h}$ of coronary occlusion and arterial reperfusion (23) in the dog.

Changes in blood viscosity. Intravascular red blood cells often appeared packed within the capillary lumens. Red blood cell packing also was noted in cat heart by Krug et al. (8), in kidney by Flores, DiBona, Beck, and Leaf (2), and by Summers and Jamison (1) and has been implicated in brain (6). This finding could indicate that a downstream obstruction to flow existed. Red blood cell packing may contribute to the no-reflow phenomenon, since rouleaux formation or localized areas of increased hematocrit can increase blood viscosity and in itself lead to stasis. Leaf has suggested that red blood cells in ischemic tissue lose their flexibility and might contribute to vascular obstruction (24). That red cell flexibility is important for normal flow is further suggested by the fact that rigid $8 \pm 1-\mu \mathrm{m}$ plastic microspheres become trapped in the microvasculature (25).

Thrombosis. In contrast to previous studies $(3,8)$, we observed occasional platelet and fibrin thrombi in areas of no reflow. It is, however, questionable that capillary thrombosis is the primary cause of the lack of blood reflow, especially since thrombi were infrequently seen by electron microscopy and were not seen by light microscopy in sections of tissue obtained 10-12 s after release of a $90-$ min occlusion or in tissue permanently ischemic for $90 \mathrm{~min}$. Further evidence that thrombosis is not the principal cause of no reflow is the fact that heparinization alone failed to prevent the no-reflow phenomenon in ischemic renal and cerebral injury $(1,6)$.

Tissue cdema. Another consistent finding in areas of no reflow was marked myocardial cell swelling. Such swelling previously has been demonstrated in ischemic myocardium (20) and is a manifestation of the loss of the capacity of the damaged cells to regulate cell volume $(19,26)$. The sarcolemmal membrane often was lifted off of the myofibrils forming large fluid-filled intracellular spaces. These large subsarcolemmal blebs sometimes appeared to compress capillaries. Only a small number of compressed capillaries could be found in any given section, but such compression is a possible mechanism 


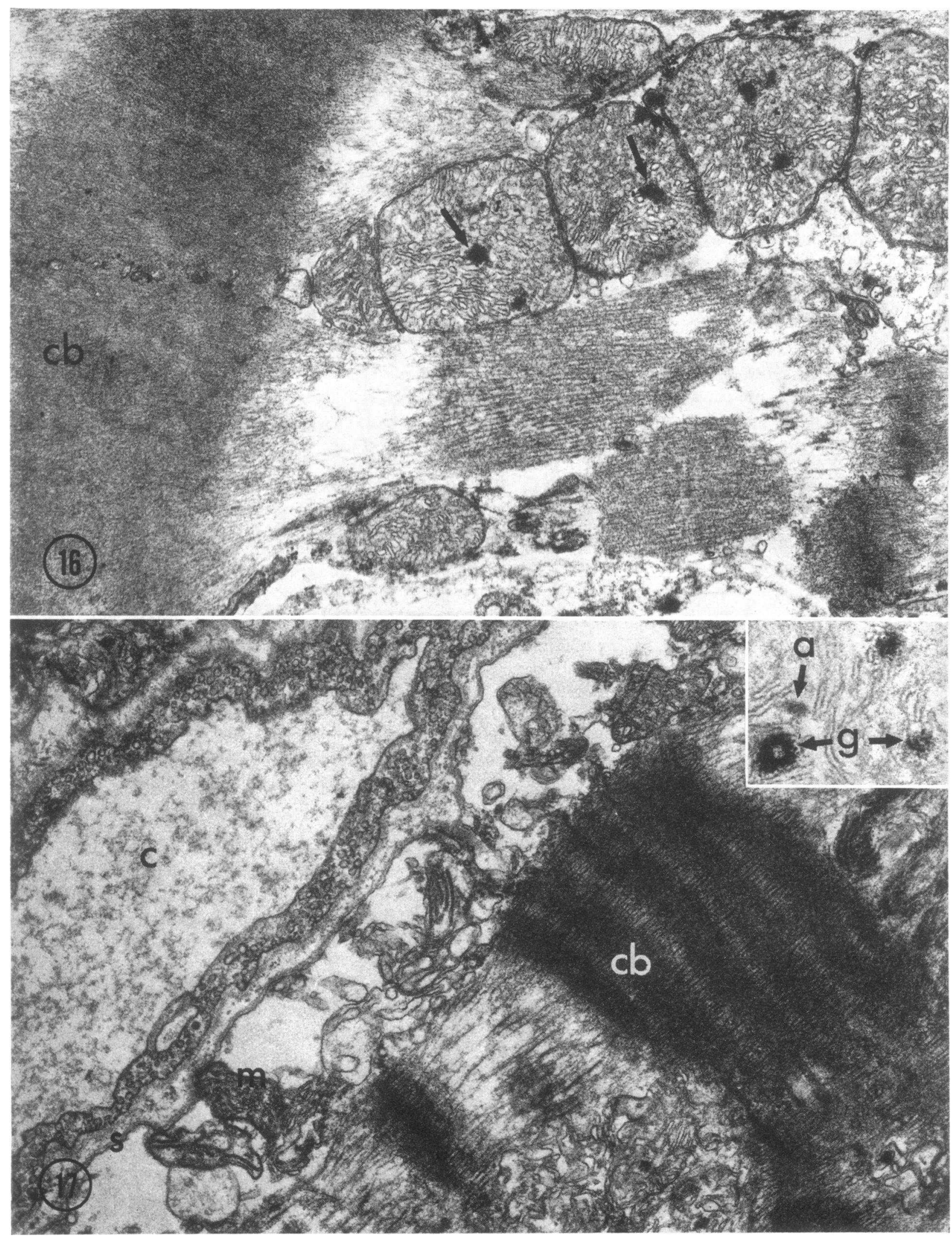

1506

R. A. Kloner, C. E. Ganote, and R. B. Jennings 
for small localized areas of increased vascular resistance. This change is somewhat analogous to the swollen astrocytes noted to be compressing capillaries in the cerebral no-reflow phenomenon (3). However, identical subsarcolemmal blebs were seen in massively-swollen, irreversibly-injured cells after $40 \mathrm{~min}$ of temporary ischemia and 2-20 min of reflow (13). Since our experiments showed that after $40 \mathrm{~min}$ of ischemia tissue was uniformly perfused with blood containing thioflavin $\mathrm{S}$, the contribution of myocardial cell swelling to the no-reflow phenomenon may be minor. On the other hand, since the thioflavin $\mathrm{S}$ method is not quantitative, increased vascular resistance with mild reduction in flow probably would not be detected in our experiments.

Electron microscopic changes in areas of reflow. In fluorescent tissue just adjacent to areas showing no reflow, myocardial cell damage was severe, but capillaries showed only minimal structural damage. In these more peripheral areas with flow, the damaged cells showed large contraction bands, whereas these were not observed in the areas of no reflow identified after $90 \mathrm{~min}$ of temporary ischemia. Previously, and in the present study, we have found contraction bands in cells localized to the periphery of areas of permanent ischemia or in irreversibly injured cells which have been exposed to arterial reperfusion. The cause of the contraction bands is unknown; they may result from the influx of extracellular $\mathrm{Ca}^{2+}$, only small amounts of which are available in the absence of flow, into damaged myocardial cells. However, mitochondria in the cells with contraction bands only had amorphous matrix densities; these cells did not contain the striking granular densities found in similar cells obtained from myocardium temporarily ischemic for $40 \mathrm{~min}$ with $2-20 \mathrm{~min}$ of reflow (Fig. 17). These granular dense bodies are characteristic of those noted with massive $\mathrm{Ca}^{2+}$ accumulation in vitro and are a consistent feature of temporary irreversible ischemic injury $(10,11,13,15)$. That $\mathrm{CaPO}_{4}$ granular dense bodies did not occur in areas of reflow after $90 \mathrm{~min}$ of ischemia probably is due to mitochondrial failure. In vitro, isolated myocardial mitochondria usually will accumulate $\mathrm{Ca}^{2+}$ after 60 min of autolysis but have lost this capacity after $120 \mathrm{~min}$ of autolysis. ${ }^{8}$ Our data indicates that $\mathrm{Ca}^{2+}$ accumulation ceases to occur in the irreversibly injured cells in the area of severe ischemia, i.e. the inner half of myocardium, sometime after 40 and before $90 \mathrm{~min}$ of ischemia have elapsed. No chemical data supporting the observation that $\mathrm{Ca}^{2+}$ accumulation does not occur at $90 \mathrm{~min}$ have been obtained.

When coronary occlusion is maintained from $90 \mathrm{~min}$ to $24 \mathrm{~h}$, the area of irreversibly injured cells increases in size to include portions of the outer half of myocardium (27). We do not know when the potential for mitochondrial calcium accumulation disappears from the more peripheral subepicardial cells which die only after longer periods of ischemia than those employed in the present study.

In summary, $40 \mathrm{~min}$ of temporary ischemia, a period known to be sufficient to cause irreversible injury to the majority of severely ischemic myocardial cells in the dog heart, causes little or no change in the vascular flow patterns observed after carbon or thioflavin $\mathrm{S}$ injections. On the other hand, $90 \mathrm{~min}$ of temporary ischemia is followed by poor or absent perfusion of large areas of the previously ischemic tissue. The results show, therefore, that the no-reflow phenomenon eventually does develop in ischemic myocardium of anesthetized open-chest dogs. Myocardial cell swelling was prominent after both 40 and 90 -min periods of ischemia, whereas capillary damage was most severe after $90 \mathrm{~min}$ of ischemia and was localized to areas of no reflow. Thus, the results suggest but do not prove that capillary damage is a major contributing factor to the development of the no-reflow phenomenon. Since cell swelling was not quantitated in this study, however, it is possible that progressive myocardial cell swelling with resultant capillary compression

\footnotetext{
${ }^{3}$ Jennings, R. B., and M. L. Hill, unpublished data.
}

FIGURE $16 \mathrm{PP}$ temporarily ischemic for $90 \mathrm{~min}$ with $20 \mathrm{~min}$ of reperfusion (from an area of reflow). Amorphous mitochondrial matrix dense bodies (arrows) and intermyofibrillar edema are prominent. Myofilaments are disorganized and a large contraction band (cb) is present. $\times 22,000$.

FIGURE 17 PP temporarily ischemic for $40 \mathrm{~min}$ with $10 \mathrm{~min}$ of coronary arterial reperfusion. A swollen myocardial cell containing a contraction band (cb) is present on the right and a capillary (c) is on the left. Note that the sarcolemma (s) is separated from the adjacent myofibrils by a large space which contains scattered mitochondria $(\mathrm{m})$. The capillary (c) is patent and the endothelial cytoplasm is filled with numerous pinocytotic vesicles. $\times 17,000$. The insert is a high-power view of a portion of a mitochrondrion from an animal given 40 min of ischemia and 20 min of reflow. It shows both amorphous (a) and granular ( $g$ ) dense bodies. The amorphous dense bodies also are found in permanently ischemic irreversibly injured cells, while the granular dense bodies only are found in such cells after arterial reperfusion. They are believed to contain calcium, probably in the form of hydroxyapatite. Although dogs with $90 \mathrm{~min}$ of ischemia and $20 \mathrm{~min}$ of reflow developed contraction bands similar to those noted in this figure, the mitochondria contained only amorphous matrix densities. Even with arterial reflow, no granular densities were formed. 
also could contribute to the no-reflow phenomenon. The role of blood viscosity also cannot be ruled out by this study.

Whether a no-reflow phenomenon occurs in man remains to be determined. The presence of a no-reflow phenomenon could have important clinical implications. Damaged tissue, which cannot be reperfused, is no longer in close contact with the circulation and presumably it would be unreachable by drug therapy or by coronary bypass surgery and should heal more slowly than tissue which is in contact with the arterial circulat:on. However, areas of no reflow in the dog are confined to the most severely ischemic myocardium in the inner ventricular wall; areas of more moderate ischemia, especially in the outer myocardial wall, retain vascular integrity and hence the potential for salvage.

\section{ACKNOWLEDGMENTS}

We wish to thank Jerome L. Hines and Carri L. Kawahara for surgical assistance; Robert L. Martinez and Rosemary Godfrey for histologic sections; and Saundra Browning, Rose Liu, Brigitte Riebe, and Susan Decker for assistance in cutting sections for electron microscopy.

This work was supported in part by National Institutes of Health grant HE 08729 and contract NHLI-72-2984.

\section{REFERENCES}

1. Summers, W. K., and R. L. Jamison. 1971. The no reflow phenomenon in renal ischemia. Lab. Invest. 25: 635-643.

2. Flores, J., D. R. DiBona, C. H. Beck, and A. Leaf. 1972. The role of cell swelling in ischemic renal damage and the protective effect of hypertonic solute. $J$. Clin. Invest. 5.1 : 118-126.

3. Chaing, J., M. Kowada, A. Ames, III, R. L. Wright, and G. Majno. 1968. Cerebral ischemia. III. Vascular changes. Am. J. Pathol. 52: 455-476.

4. Majno, G., A. Ames, III, J. Chaing, and R. L. Wright. 1967. No reflow after cerebral ischemia. Lancet. 2: 569-570.

5. Kowada, M., A. Ames, III, G. Majno, and R. L. Wright. 1968. Cerebral ischemia. I. An improved experimental method for study; cardiovascular effects and demonstration of an early vascular lesion in the rabbit. J. Neurosurg. $20: 150-157$.

6. Fischer, E. G., and A. Ames, III. 1972. Studies on mechanisms of impairment of cerebral circulation following ischemia: effect of hemodilation and perfusion pressure. Stroke J. Cereb. Circ. 3: 538-542.

7. Willms-Kretschmer, K., and G. Majno. 1969. Ischemia of the skin. Electron microscopic study of vascular injury. Am. J. Pathol. 54: 327-343.

8. Krug, A., W. M. de Rochemont, and G. Korb. 1966. Blood supply of the myocardium after temporary coronary occlusion. Circ. Res. 19: 57-62.

9. Willerson, J. T., W. J. Powell, Jr., T. E. Guiney, J. J. Stark, C. A. Sanders, and A. Leaf. 1972. Improvement in myocardial function and coronary blood flow in ischemic myocardium after mannitol. J. Clin. Invest. 51 : 2989-2998
10. Shen, A. C., and R. B. Jennings. 1972. Myocardial calcium and magnesium in acute ischemic injury. $\mathrm{Am}$. J. Pathol. 67 : 417-433.

11. Shen, A. C., and R. B. Jennings. 1972. Kinetics of calcium accumulation in acute myocardial ischemic injury. Am. J. Pathol. 67: 441-452.

12. Kloner, R. A., C. E. Ganote, K. A. Reimer, and R. B. Jennings. 1974. Coronary flow during acute myocardial ischemia. Fed. Proc. 33: 592. (Abstr.)

13. Kloner, R. A., C. E. Ganote, D. H. Whalen, Jr., and R. B. Jennings. 1974. Effect of a transient period of ischemia on myocardial cells II. Fine structure during the first few minutes of reflow. Am. J. Pathol. 74: 399-413.

14. Caulfield, J., and B. Klionsky. 1959. Myocardial ischemia and early infarction: an electron microscopic study. Am. J. Pathol. 35 : 489-501.

15. Herdson, P. B., R. B. Jennings, and H. M. Sommers. 1965. A comparative study of the fine structure of normal and ischemic dog myocardium with special reference to early changes following temporary occlusion of a coronary artery. Am. J. Pathol. 46: 367-386.

16. Franklin, W., C. E. Ganote, and R. B. Jennings. 1974. Blood reflow after renal ischemia. Effects of hypertonic mannitol on reflow and tubular necrosis after transient ischemia in the rat. Arch. Pathol. In press.

17. Schlegel, J. U. 1949. Demonstration of blood vessels and lymphatics with a fluorescent dye in ultraviolet light. Anat. Rec. 105 : 433-443.

18. Dohrmann, G. J., K. M. Wick, and P. C. Bucy. 1973 Spinal cord blood flow patterns in experimental traumatic paraplegia. J. Neurosurg. 38: 52-58.

19. Whalen, D. A., Jr., D. G. Hamilton, C. E. Ganote and R. B. Jennings. 1974. Effect of a transient period of ischemia on myocardial cells. I. Effects on cell volume regulation. Am. J. Pathol. 74: 381-397.

20. Jennings, R. B., J. H. Baum, and P. B. Herdson. 1965. Fine structural changes in myocardial ischemic injury. Arch. Pathol. 79: 135-143.

21. Zollinger, H. U. 1948. Cytologic studies with the phase microscope I. The formation of "blisters" on cells in suspension (potocytosis) with observations on the nature of the cellular membrane. Am. J. Pathol. 24: 545567.

22. Goldberg, B., and H. Green. 1959. The cytotoxic action of immune gamma globulin and complement on Krebs ascites tumor cells I. Ultrastructural studies. J. Exp. Med. 109: 505-510.

23. Lang, T-W., E. Corday, H. Gold, S. Meerbaum, S Rubins, C. Costantini, S. Hirose, J. Osher, and V. Rosen. 1974. Consequences of reperfusion after coronary occlusion. Effects on hemodynamic and regional myocardial metabolic function. Am. J. Cardiol. 33: 69-81.

24. Leaf, A. 1973. Cell swelling: a factor in ischemic tissue injury. Circulation. 48: 455-458.

25. Buckberg, G. D., J. C. Luck, D. B. Payne, J. I. E Hoffman, J. P. Archie, and D. E. Fixler. 1971. Some sources of error in measuring regional blood flow with radioactive microspheres. J. Appl. Physiol. 31: 598-604.

26. Leaf, A. 1970. Regulation of intracellular fluid volume and disease. Am. J. Med. 49: 291-295.

27. Rasmussen, M. M., K. A. Reimer, and R. B. Jennings. 1974. Propranolol therapy in experimental myocardia ischemia. Fed. Proc. 33: 591. (Abstr.) 